\title{
Interstellar Carbon Dust
}

\author{
Emmanuel Dartois \\ Institut des Sciences Moléculaires d'Orsay (ISMO), UMR8214, CNRS-Université de Paris-Sud, \\ Université Paris-Saclay, Bât 520, Rue André Rivière, F-91405 Orsay, France; emmanuel.dartois@u-psud.fr
}

Received: 29 September 2019; Accepted: 12 November 2019; Published: 2 December 2019

\begin{abstract}
In the ranking of cosmic abundance of the elements, carbon is the second element, after oxygen, able to form multiple bonds propagating the formation of a network, thus playing an essential role in the formation of nanometer- to micrometer-sized interstellar dust grains. Astrophysical spectroscopic observations give us remote access to the composition of carbonaceous and organic interstellar grains. Their presence and abundances from spectroscopic observations and the phases of importance for the Galactic carbon budget are considered in this article.
\end{abstract}

Keywords: interstellar medium; dust grains; organic matter

\section{Introduction}

The interstellar space is filled with gas and dust grains. Interstellar carbonaceous large molecules and dust grains hold a large fraction of the available carbon abundance in the Galaxy. Various forms of carbon are observed and measured, the most recent allotropes observed are space fullerenes. The different potential carriers are traced via spectroscopic specific bands observed in emission and/or absorption. They are related to various allotropic forms of carbonaceous dust present in the interstellar medium and around young and old stars, ranging from purely carbon forms to carbon dust containing hydrogen and heteroatoms. The observations are compared to experiments conducted for identification and interpretation of these astronomical spectra. The several observed forms of carbon in space include (nano-)diamonds, fullerenes, polyaromatic structures, amorphous carbon and their hydrogenated amorphous carbon forms (generally named HAC in astrophysics and a-C:H in physics), as well as by extension the organic matter that originates from the energetic processing of ice mantles in dense cloud regions of the Galaxy and/or from the possible condensation in dense and chemically-rich stellar wind around evolved stars [1,2]. These dust grains are immersed in harsh astrophysical environments including vacuum ultraviolet photons and high energy accelerated particles (the cosmic rays), thermal evolution and will thus be processed.

In many astrophysical environments where they reside, the question of their contribution to the enrichment of the medium with complex organic molecules by top down chemistry (i.e., the destruction in smaller entities of initially very large size molecules or even grains) must thus be raised and assessed.

\section{Observations of Interstellar Carbons}

The vast majority of the information on interstellar space comes from spectroscopy for the obvious reason of the distance to the studied astrophysical objects. The solar system is the exception, for which samples can be examined by space probes carrying more than spectroscopic instruments or in favourable cases, extraterrestrial matter can be studied in the laboratory, for example, meteorites and interplanetary dust particles captured by Earth or sample return missions to comets and asteroids. The physico chemical composition of carbon based large molecules and dust grains can be observed either in emission or in absorption, depending on the astrophysical environment, size of the molecules and excitation source. The main components observed in emission in the Galaxy consist 
of emission bands in the mid infrared, peaking around 3.3, 6.2, 7.7, 8.6, 11.3, $12.7 \mu \mathrm{m}$, characteristic of a polyaromatic component, the so-called aromatic infrared bands (AIBs) or Polycyclic aromatic hydrocarbon hypothesis ("PAHs") (Figure 1, lower right). Some specific environments also show emission spectra with an aliphatic character, in addition to the polyaromatic structure. Broad continua emission is also often present under these bands and extending to longer wavelength. In absorption, characteristic absorptions are observed in the mid infrared at about 3.4, 6.85 and 7.2 $\mu \mathrm{m}$ along long distant lines of sight (Figure 1, upper left), characteristic of hydrogenated amorphous carbons. Another spectroscopic signature occurs in the vacuum ultraviolet, with a broad absorption band around $217.5 \mathrm{~nm}$, accompanied by a rise in the deep ultraviolet. These absorptions are related to the electronic absorption of carbon based species.

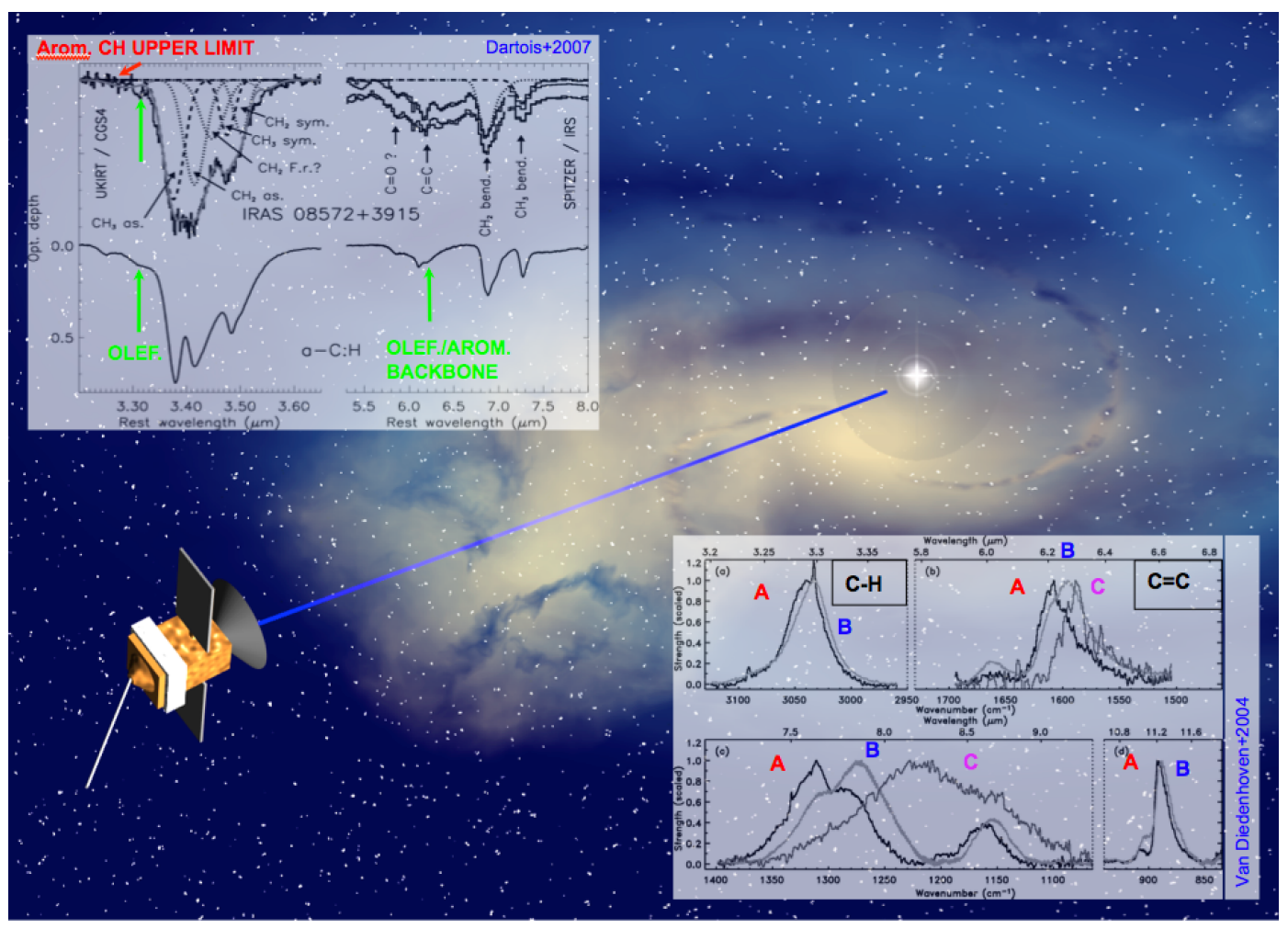

Figure 1. Observations along lines of sight toward the Galactic center or, even looking at other galaxies, allow one to probe interstellar dust. Using a bright distant infrared background source, the diffuse interstellar medium dust grain infrared signatures of hydrogenated amorphous carbons can be probed in absorption (e.g., shown in the upper left panel, compared to laboratory experiments). Another component of the diffuse medium, aromatic infrared bands can be observed in emission in many distinct lines of sight (e.g., lower right signatures). See References [3-5] for details of the inserts. Inserts from Dartois et al., A\&A. 463, p637, 2007, reproduced with permission @ ESO, and from Van Diedenhoven et al., ApJ 611, p933, 2004, reproduced with permission @ AAS.

\section{1. (Nano-)Diamonds}

The existence of diamonds in interstellar space was postulated after the detection of small diamonds of presolar origin in meteorite extracts, with abundances sometimes almost approaching the part per thousands in some carbonaceous chondrites meteorites. Some of these diamond phases are truly attributed to a presolar origin based notably on their isotopic anomalies, others may be readily explained by high temperature and/or shock processing of carbon containing parent bodies or meteorites during solar system evolution [6-14]. The first detection of nanodiamonds in space have been performed by Reference [15] and unambiguously confirmed by comparing emission spectra of the dust grains evolving in circumstellar environments around the intermediate mass (a few $\mathrm{M}_{\odot}$ ) Herbig Ae-Be stars to laboratory spectra [16]. These authors identified specific infrared emission bands 
at 3.43 and $3.53 \mu \mathrm{m}$ attributed to the vibrational $\mathrm{CH}$ elongation modes of hydrogenated diamond nanoparticle surfaces. These interstellar medium (ISM) nanodiamonds spectra are different from the one recorded for meteoritic extracted nanodiamonds [17]. Laboratory approaches to reproduce the observed ratios of the ISM observed bands have followed a top down (nanostructures are synthesized starting from big units, reducing their size and following the spectral evolution associated) or bottom up approach (starting from the molecular world, following the spectral evolution using small diamondoid molecules that are grown in size and measured experimentally and/or explored bigger and bigger also by quantum calculation) to constrain the sizes of these remotely observed hydrogenated diamond terminations $[16,18,19]$. The top down nano-diamond approach led to an estimate of relatively large sizes for the interstellar nano-diamonds and they were found to be related to the non relaxed surfaces for nano-diamonds in the experiments for sizes below $35 \mathrm{~nm}$. The bottom-up molecular approach, combined with density functional calculations demonstrated that the observed intensity ratio of the two bands observed at $3.53 \mu \mathrm{m}$ and $3.43 \mu \mathrm{m}$ would imply a much lower size, with about 136 carbon atoms $(\sim 1.5 \mathrm{~nm})$. A few sources display the characteristic emissions attributed to $\mathrm{CH}$ diamond terminations [20]. An observational survey of about thirty lines of sight with expected similar astrophysical environments have been conducted by Reference [21], with no additional detections of the presence of these nanodiamond emission bands. Several observations have also succeeded in resolving spatially these emission bands [22-24] to understand the underlying physical conditions for their formation. In resolved sources they appear relatively close to the stars, closer than other carbon dust emission bands associated with polyaromatic materials. Observations at higher spatial resolutions will help in better defining where these diamonds reside and emit, and put additional constraints on their sizes. Their detection in only a handful of sources questions their overall importance in the carbon budget of the Galaxy. These interstellar nanodiamonds are among the best identified spectroscopic features for interstellar dust emission bands, from the spectroscopic point of view and are a potential probe of very specific carbon allotrope forming environments. Pushing the detection capabilities of space telescopes will assess to what extent these nanodiamonds should be considered as widespread.

\subsection{Fullerenes}

The existence of fullerenes in space has been postulated since their discovery by Reference [25]. A long search started in the visible to the infrared range. Reference [26] found that two interstellar absorption bands observed in lines of sight probing the diffuse intertellar medium almost matched to $\mathrm{C}_{60}^{+}$absorptions recorded by Reference [27] in matrix isolation spectroscopy experiments. Reference [28], looking at absorption in the optical along lines of sight toward distant massive stars, probing the diffuse interstellar medium, could not find electronic absorptions of neutral $\mathrm{C}_{60}$, estimating that $\mathrm{C}_{60}^{+} / \mathrm{C}_{60} \geq 100$. Reference [29] reported upper limits on the detection of both species in emission in the vibrational infrared. The first detection of $\mathrm{C}_{60}$ (single molecular species in vacuum) was reported by $[30,31]$ through the main vibrationally active modes of $\mathrm{C}_{60}$, spatially resolved in emission in a reflection nebula. Reference [32] reported the detection of $C_{60}$ and $C_{70}$ in emission around a protoplanetary nebula particularly poor in hydrogen. In the infrared, it has been reported in many other astrophysical lines of sight [33-49], mainly in protoplanetary nebulae sources, but also many other objects such as reflection nebulae, Asymptotic Giant Branch (AGB) and Herbig Ae/Be stars. In recent years, ion trap action spectroscopy allowed the measurement of almost unperturbed free flying $\mathrm{C}_{60}^{+}$molecule electronic transitions in the laboratory [50,51]. They allowed the assignment of diffuse interstellar bands to $\mathrm{C}_{60}^{+}$. Other fullerene cations have been measured in the laboratory [52-54], and investigated observationally but their lower intensity makes them often too difficult to observe astronomically. From laboratory experiment [55] the intrinsic strength of the transitions can be estimated. The corresponding amount of carbon locked in $\mathrm{C}_{60}$ or $\mathrm{C}_{60}^{+}$is relatively low, of the order of a few $10^{-5}$ to $10^{-4}$ of the carbon cosmic abundance $[56,57]$. 


\subsection{Graphite}

Graphite is often referred to as an interstellar component. Its presence in the interstellar medium has been supported initially by the ultraviolet extinction observations (e.g., References [58-61]) and the hypothesis reinforced by the finding of some preserved presolar graphite grains in some meteorites. It has been used to fit the ultraviolet extinction curve observed in the diffuse medium. The model requirements to obtain a good fit imply a precise knowledge on the optical constants to be used [62]. Critical adjustments on the aspect ratio of pure graphite grains, as well as their size distribution are required to adjust the extinction curves [63-69] and it is thus considered by some authors as too demanding, a too narrow parameter space with respect to the diversity of environments expected in astrophysics. Many other carbon based material combinations can produce an extinction in the same wavelength range, offering alternatives to "pure" graphite grains [70-82]. Graphite is still used in some astronomical models as a template carbon base, sometimes denominated "interstellar graphite" [83] when it is adjusted based on observed interstellar extinction curves.

\subsection{Silicon Carbide}

Among Carbides, one of the most abundant expectation in the ISM is based on Silicon Carbide. While it is highly discussed in the meteorites community since a long time (see, e.g., Reference [84,85]), and some $\mathrm{SiC}$ dust particles are observed in emission or absorption around some C-rich stars (e.g., References [86-89]), it has not been detected in the diffuse ISM so far, whereas given the strength of the $\mathrm{SiC}$ vibrational mode, observable at $11.3 \mu \mathrm{m}$, in the shoulder of interstellar silicates, if present in abundance it should be observed, if it represents at least a few percents in abundance with respect to the silicates (e.g., References $[90,91])$. The reason of its absence in the diffuse ISM is the subject of a debate, including discussions on its survival after its formation in the envelopes of C-rich stars. Some observational constraints should be further pushed in the near future, but the vast majority of the silicon atoms seem engaged in the formation of silicates rather than $\mathrm{SiC}$ (e.g., Reference [92]).

\subsection{The PAH Hypothesis}

Among the most popular carbon based structures in space are the so-called Polycyclic Aromatic Hydrocarbons (PAHs). PAH refers to the infrared fluorescence of large free flying PAH-like entities, upon absorption and relaxation of a single energetic UV photon. This name follows the first description by References [93,94] to account for infrared emission bands observed at 3.3, 6.2, 7.7, 8.6, and $11.3 \mu \mathrm{m}$ in many astronomical lines of sight, on the basis of laboratory PAH photophysics. This terminology is now part of a common language of astronomers, but should be more adequately stated as the "Polycyclic Aromatic Hydrocarbons hypothesis." A more suited term to name these emissions is probably Aromatic Infrared Bands (AIBs), as it is clear that these astrophysical bands arise in a polyaromatic system. However, such emissions are still unidentified with a definite carrier. The observed profile variations from source to source of these emission bands have been categorized [5,95] and named of classes $\mathcal{A}, \mathcal{B}, \mathcal{C}$. More recently a $\mathcal{D}$ class has been added [96]. The class $\mathcal{A}$ band positions display a highly aromatic character, whereas evolving toward the $\mathcal{C}$ and $\mathcal{D}$ classes, the emission bands also include some aromatic/aliphatic mixed character as seen in observations and experiments [96-103]. From an observational point of view, the class $\mathcal{A}$ emission bands are widespread and seen in many different observed source lines of sight, indicating that these AIBs carriers are present in distinct astrophysical environments and relatively easily excited by photons. If the changing profiles are associated with an evolution of the chemical composition of the carriers, the ubiquitous presence of class $\mathcal{A}$ emission is also attributed to a fluorescence mechanism, which is independent of the photon flux, and thus the distance to the photon source. Some of the class $\mathcal{C}$ and $\mathcal{D}$ emissions may be observed less frequently because they either are less represented in abundance, and/or are related to a thermal emission mechanism, active only relatively close to the photon sources. The observation statistics of 
the different classes of AIBs is thus weighted by the variations of the astrophysical environments but also their intrinsic emitting mechanism efficiency.

\subsection{Amorphous Carbon}

There are not so many studies on the spectroscopy of astrophysical objects displaying amorphous (hydrogen-free) carbon dust. Such dust grains are injected into the diffuse medium, but then are difficult to observe, as generally they are only seen close to the stellar ejecta through a featureless blackbody-like continuum emission [86,104-107]. Once injected, such dust could be hydrogenated later, during its journey (e.g., References $[97,108-110]$ ) and irradiation in astrophysical atomic hydrogen dominated environments [111,112].

Hydrogenated amorphous carbons form another important carbonaceous species in the interstellar medium that is often overlooked because of the weaker intrinsic absorption features used to probe them. They however represent one of the major reservoirs of carbon based dust in galaxies. Observed for a long time from the ground through an absorption band at $3.4 \mu \mathrm{m}$ in the direction of the Galactic center lines of sight, against bright infrared background sources [113], its observability requires a significant amount of column density, generally associated with distant lines of sight. The profile of the band can be assigned to methyl and methylene $\left(\mathrm{sp}^{3} \mathrm{CH}_{3}\right.$ and $\left.\mathrm{CH}_{2}\right)$ elongation modes in a more complex dust grain [114]. Several suggestions have been made on the macromolecular structure supporting the spectroscopic observations. They evolved with time as the constraints from satellite observations yield data over a broader wavelength range, discarding some of the very first thoughts based on the $3.4 \mu \mathrm{m}$ feature profile only [115-117]. The hydrogenated amorphous carbon signature is observed ubiquitously in the interstellar medium of extragalactic sources $[3,4,118-120]$.

\section{Evolution and Interaction with the Environment}

The detection of large carbon molecules/radicals at the interfaces between the diffuse interstellar medium and dense cloud condensations is difficult to reproduce with astrophysical models including only gas phase chemistry. This questions the eventual proportions inherited from the destruction of carbonaceous dust grains, that is, the evolution and interaction with the environment of these grains [121-125].

Astronomical observations results from an equilibrium between destruction and production of species. The AIBs (PAHs) and a-C:H at the interface of Photon Dominated Regions (PDRs), transition regions between a diffuse (a few hydrogen per cubic centimetre) to denser medium (typically above a thousand hydrogen molecules or atoms per cubic centimetre) and exposed to harsh VUV photons generating an active photochemistry) are invoked to explain the observation of numerous hydrocarbon molecule detections and suggest the possibility of a top-down chemistry in such environments. These environments as well as the diffuse medium are also exposed to high energy atomic ions interacting with the astrophysical solids. Some laboratory experiments aim at constraining the observation of the evolution of such carbonaceous species within the ISM and at PDRs interfaces with denser regions and interactions with the radiative environment they are immersed in References [78,126-132]. A typical setup to monitor the species produced by interaction with astrophysical environments is shown in Figure 2. The laboratory photolytic and/or radiolytic rates, discussed in the above cited references, can be implemented in astrophysical models and provide a better match to observations of the gas phase abundances, in particular for large carbonaceous species, as well as evolution time scales that are in accordance with the expected diffuse-to-dense interface advection, that is, the speed at which the dense cloud interface is moving because of the strong UV field nibbling the dense interface. Both cosmic rays and VUV photons release carbonaceous species, feeding the ladder of large carbon species observed in astronomy. The laboratory experiments support the production of species from the solid phase and the evolution toward reactive and bigger species [133] detection is an active field of research. 


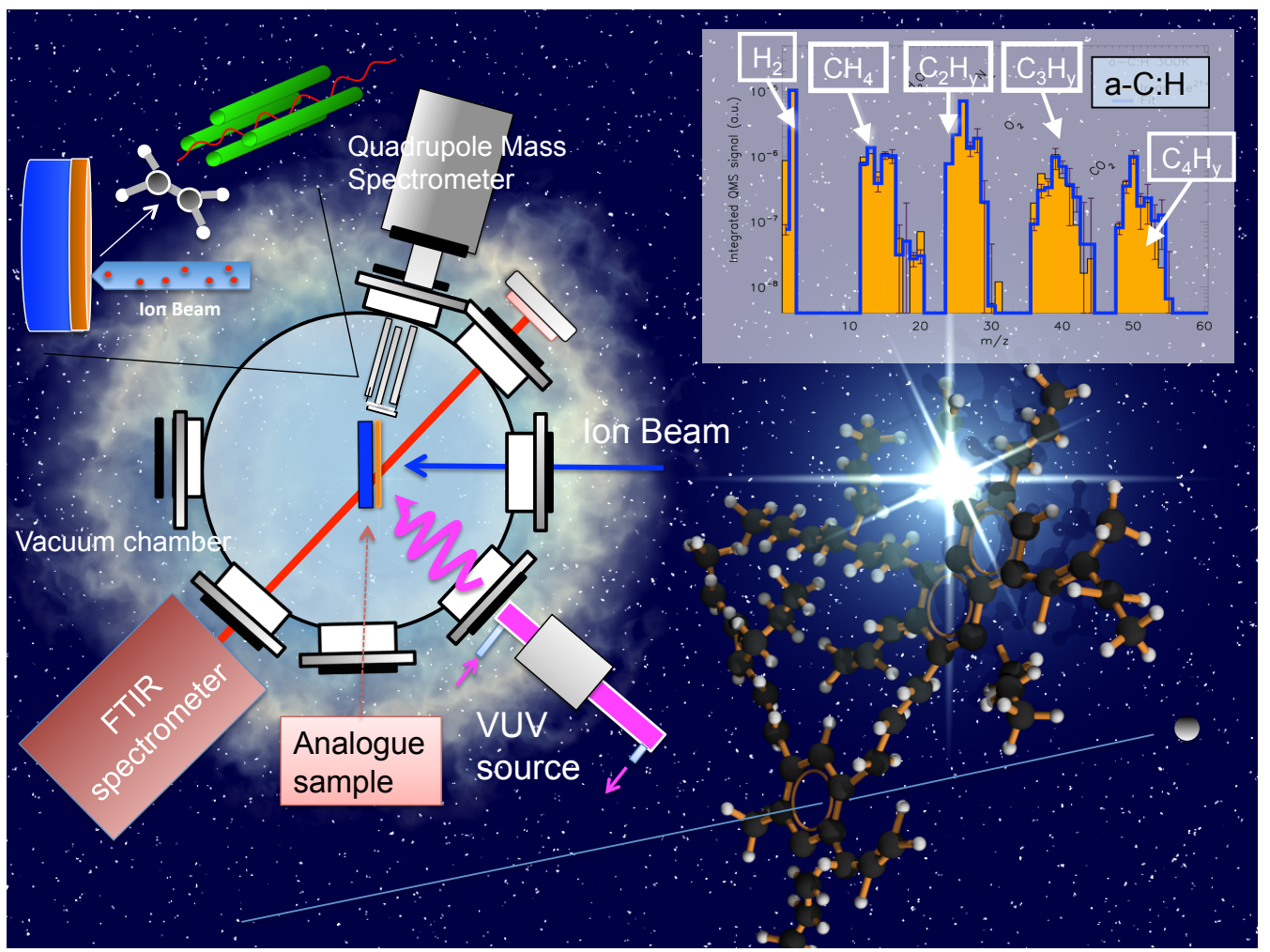

Figure 2. Typical scheme of experiments aiming at following the evolution of a dust analogue subjected to either ion irradiation simulating cosmic rays [126] or energetic VUV photons [127] from the interstellar radiation field. An interstellar analogue is placed at the center of the chamber, maintained at interstellar low temperatures and the evolution and products of the photolysis and or radiolysis are monitored both in the solid phase and the gas phase. Insert QMS spectrum from Dartois et al., A\&A. 599, A130, p6, 2017, reproduced with permission (c) ESO.

\section{Which Carbonaceous Solids Are Ingredients for Interstellar Models?}

Observational biases, underlying emission mechanisms and spectroscopic contrasts favour the visibility of some of the carbonaceous dust components. When one examine the mass balance, based on the previously discussed astronomical observational spectroscopic constraints, neither (nano-)diamonds nor fullerenes seem abundant enough to validate the claim that they are major carbonaceous interstellar dust grains components. However, their presence reflects important evolutionary paths in the interstellar physico-chemistry, and they are of relevance to provide constraints on the astrochemistry at work. Focusing on the vast majority of interstellar grains, that is, the most abundant ones, then (hydrogenated) amorphous carbons and AIBs (polyaromatic) carriers dominate, carrying a large fraction of the available cosmic carbon. The carriers of the AIBs constitute an important part of astrophysical models, as they must account for the emission bands observed in the mid infrared. The requirements in models are around five percent of the available cosmic carbon abundance [134-142]. The hydrogenated amorphous carbon absorptions trace a material that represents an abundance higher than what the AIBs emission bands imply. The exact abundance will vary according to the dust grain carrier network adopted in the experiments and modeling, but are not less than a few percent and maybe up to about $40 \%$ of the available cosmic carbon abundance [3,143-146]. Some models are now including these alternative forms of carbon including polyaromatic and/or hydrogenated amorphous carbons, gradually abandoning models dominated by graphite as a proxy for carbon dust grains [147-149]. 
Funding: This work was supported by the Programme National "Physique et Chimie du Milieu Interstellaire" (PCMI) of CNRS/INSU with INC/INP co-funded by CEA and CNES.

Conflicts of Interest: The author declares no conflict of interest.

\section{Abbreviations}

The following abbreviations are used in this manuscript:

$\begin{array}{ll}\text { AIBs } & \text { Aromatic Infrared Bands } \\ \text { PDR } & \text { Photon or Photodissociation dominated region } \\ \text { ISM } & \text { Interstellar Medium } \\ \text { PAHs } & \text { Polycyclic aromatic Hydrocarbons }\end{array}$

\section{References}

1. Dartois, E.; Alata, I.; Engrand, C.; Brunetto, R.; Duprat, J.; Pino, T.; Quirico, E.; Remusat, L.; Bardin, N.; Briani, G.; et al. Interstellar and interplanetary solids in the laboratory. Bull. Soc. R. Sci. Liege 2015, 84, 7-14. [CrossRef]

2. Kwok, S. Complex organics in space from Solar System to distant galaxies. Astron. Astrophys. Rev. 2016, 24, 8. [CrossRef]

3. Dartois, E.; Muñoz-Caro, G.M. Carbonaceous dust grains in luminous infrared galaxies. Spitzer/IRS reveals a-C:H as an abundant and ubiquitous ISM component. Astron. Astrophys. 2007, 476, 1235-1242. [CrossRef]

4. Dartois, E.; Geballe, T.R.; Pino, T.; Cao, A.-T.; Jones, A.; Deboffle, D.; Guerrini, V.; Bréchignac, P.; D'Hendecourt, L. IRAS 08572+3915: Constraining the aromatic versus aliphatic content of interstellar HACs. Astron. Astrophys. 2007, 463, 635-640. [CrossRef]

5. Van Diedenhoven, B.; Peeters, E.; van Kerckhoven, C.; Hony, S.; Hudgins, D.M.; Allamandola, L.J.; Tielens, A.G.G.M. The Profiles of the 3-12 Micron Polycyclic Aromatic Hydrocarbon Features. Astrophys. J. 2004, 611, 928-939. [CrossRef]

6. Hanneman, R.E.; Strong, H.M.; Bundy, F.P. Hexagonal Diamonds in Meteorites: Implications. Science 1967, 155, 995-997. [CrossRef]

7. Vdovykin, G.P. Ureilites. Space Sci. Rev. 1970, 10, 483-510. [CrossRef]

8. Berkley, J.L.; Taylor, G.J.; Keil, K.; Harlow, G.E.; Prinz, M. The nature and origin of ureilites. Geochim. Cosmochim. Acta 1980, 44, 1579-1597. [CrossRef]

9. Lewis, R.S.; Ming, T.; Wacker, J.F.; Anders, E.; Steel, E. Interstellar diamonds in meteorites. Nature 1987, 326, 160-162. [CrossRef]

10. Amari, S.; Lewis, R.S.; Anders, E. Interstellar grains in meteorites: I. Isolation of SiC, graphite and diamond; size distributions of $\mathrm{SiC}$ and graphite. Geochim. Cosmochim. Acta 1994, 58, 459-470. [CrossRef]

11. Zinner, E. Stellar Nucleosynthesis and the Isotopic Composition of Presolar Grains from Primitive Meteorites. Annu. Rev. Earth Planet. Sci. 1998, 26, 147-188. [CrossRef]

12. Hill, H.G.M.; Jones, A.P.; D'Hendecourt, L.B. Diamonds in carbon-rich proto-planetary nebulae. Astron. Astrophys. 1998, 336, L41-L44.

13. Clayton, D.D.; Nittler, L.R. Astrophysics with Presolar Stardust. Annu. Rev. Astron. Astrophys. 2004, 42, 39-78. [CrossRef]

14. Heck, P.R.; Stadermann, F.J.; Isheim, D.; Auciello, O.; Daulton, T.L.; Davis, A.M.; Elam, J.W.; Floss, C.; Hiller, J.; Larson, D.J.; et al. Atom-probe analyses of nanodiamonds from Allende. Meteor. Planet. Sci. 2014, 49, 453-467. [CrossRef]

15. Guillois, O.; Ledoux, G.; Reynaud, C. Diamond Infrared Emission Bands in Circumstellar Media. Astrophys. J. Lett. 1999, 521, L133-L136. [CrossRef]

16. Chang, H.C.; Lin, J.-C.; Wu, J.-Y.; Chen, K.-H. Infrared Spectroscopy and Vibrational Relaxation of CH, and CD, Stretches on Synthetic Diamond Nanocrystal Surfaces. J. Phys. Chem. 1995, 99, 11081-11088 [CrossRef]

17. Mutschke, H.; Andersen, A.C.; Jäger, C.; Henning, T.; Braatz, A. Optical data of meteoritic nano-diamonds from far-ultraviolet to far-infrared wavelengths. Astron. Astrophys. 2004, 423, 983-993. [CrossRef] 
18. Chen, Y.-R.; Chang, H.-C.; Cheng, C.-L.; Wang, C.-C.; Jiang, J.C. Size dependence of CH stretching features on diamond nanocrystal surfaces: Infrared spectroscopy and density functional theory calculations. J. Chem. Phys. 2003, 119, 10626-10632. [CrossRef]

19. Pirali, O.; Vervloet, M.; Dahl, J.E.; Carlson, R.M.K.; Tielens, A.G.G.M.; Oomens, J. Infrared Spectroscopy of Diamondoid Molecules: New Insights into the Presence of Nanodiamonds in the Interstellar Medium. Astrophys. J. 2007, 661, 919-925. [CrossRef]

20. Van Kerckhoven, C.; Tielens, A.G.G.M.; Waelkens, C. Nanodiamonds around HD 97048 and Elias 1. Astron. Astrophys. 2002, 384, 568-584. [CrossRef]

21. Acke, B.; van den Ancker, M.E. A survey for nanodiamond features in the 3 micron spectra of Herbig Ae/Be stars. Astron. Astrophys. 2006, 457, 171-181. [CrossRef]

22. Habart, E.; Testi, L.; Natta, A.; Carbillet, M. Diamonds in HD 97048: A Closer Look. Astrophys. J. Lett. 2004, 614, L129-L132. [CrossRef]

23. Goto, M.; Henning, Th.; Kouchi, A.; Takami, H.; Hayano, Y.; Usuda, T.; Takato, N.; Terada, H.; Oya, S.; Jäger, C.; et al. Spatially Resolved $3 \mu \mathrm{m}$ Spectroscopy of Elias 1: Origin of Diamonds in Protoplanetary Disks. Astrophys. J. 2009, 693, 610-616. [CrossRef]

24. Boutéraon, T.; Habart, E.; Ysard, N.; Jones, A.P.; Dartois, E.; Pino, T. Carbonaceous nano-dust emission in proto-planetary discs: The aliphatic-aromatic components. Astron. Astrophys. 2019, 623, A135. [CrossRef]

25. Kroto, H.W.; Heath, J.R.; Obrien, S.C.; Curl, R.F.; Smalley, R.E. C60: Buckminsterfullerene. Nature 1985, 318, 162-163. [CrossRef]

26. Foing, B.H.; Ehrenfreund, P. Detection of two interstellar absorption bands coincident with spectral features of $\mathrm{C}_{60}{ }^{+}$. Nature 1994, 369, 296-298. [CrossRef]

27. Fulara, J.; Jakobi, M.; Maier, J.P. Electronic and infrared spectra of $\mathrm{C}_{60}^{+}$and $\mathrm{C}_{60}^{-}$in neon and argon matrices. Chem. Phys. Lett. 1993, 211, 227-234. [CrossRef]

28. Herbig, G.H. The Search for Interstellar C 60 . Astrophys. J. 2000, 542, 334-343. [CrossRef]

29. Moutou, C.; Sellgren, K.; Verstraete, L.; Léger, A. Upper limit on $\mathrm{C}_{60}$ and $\mathrm{C}_{60}(+)$ features in the ISO-SWS spectrum of the reflection nebula NGC 7023. Astron. Astrophys. 1999, 347, 949-956.

30. Sellgren, K.; Werner, M.W.; Ingalls, J.G. The 5-15 Micron Spectrum of Reflection Nebulae as a Probe for Fullerenes. Am. Astron. Soc. Meet. Abstr. 2009, 214, 402.12.

31. Sellgren, K.; Werner, M.W.; Ingalls, J.G.; Smith, J.D.T.; Carleton, T.M.; Joblin, C. C60 in Reflection Nebulae. Astrophys. J. Lett. 2010, 722, L54-L57. [CrossRef]

32. Cami, J.; Bernard-Salas, J.; Peeters, E.; Malek, S.E. Detection of $\mathrm{C}_{60}$ and $\mathrm{C}_{70}$ in a Young Planetary Nebula. Science 2010, 329, 1180. [CrossRef] [PubMed]

33. García-Hernández, D.A.; Manchado, A.; García-Lario, P.; Stanghellini, L.; Villaver, E.; Shaw, R.A.; Szczerba, R.; Perea-Calderón, J.V. Formation of Fullerenes in H-containing Planetary Nebulae. Astrophys. J. Lett. 2010, 724, L39-L43. [CrossRef]

34. Clayton, G.C.; de Marco, O.; Whitney, B.A.; Babler, B.; Gallagher, J.S.; Nordhaus, J.; Speck, A.K.; Wolff, M.J.; Freeman, W.R.; Camp, K.A.; et al. The Dust Properties of Two Hot R Coronae Borealis Stars and a Wolf-Rayet Central Star of a Planetary Nebula: In Search of a Possible Link. Astron. J. 2011, 142, 54. [CrossRef]

35. Gielen, C.; Cami, J.; Bouwman, J.; Peeters, E.; Min, M. Carbonaceous molecules in the oxygen-rich circumstellar environment of binary post-AGB stars. $\mathrm{C}_{60}$ fullerenes and polycyclic aromatic hydrocarbons. Astron. Astrophys. 2011, 536, A54. [CrossRef]

36. García-Hernández, D.A.; Iglesias-Groth, S.; Acosta-Pulido, J.A.; Manchado, A.; García-Lario, P.; Stanghellini, L.; Villaver, E.; Shaw, R.A.; Cataldo, F. The Formation of Fullerenes: Clues from New $\mathrm{C}_{60}$, $\mathrm{C}_{70}$, and (Possible) Planar $\mathrm{C}_{24}$ Detections in Magellanic Cloud Planetary Nebulae. Astrophys. J. Lett. 2011, 737, L30. [CrossRef]

37. Zhang, Y.; Kwok, S. Detection of $\mathrm{C}_{60}$ in the Protoplanetary Nebula IRAS 01005+7910. Astrophys. J. 2011, 730, 126. [CrossRef]

38. García-Hernández, D.A.; Villaver, E.; García-Lario, P.; Acosta-Pulido, J.A.; Manchado, A.; Stanghellini, L.; Shaw, R.A.; Cataldo, F. Infrared Study of Fullerene Planetary Nebulae. Astrophys. J. 2012, 760, 107. [CrossRef]

39. Bernard-Salas, J.; Cami, J.; Peeters, E.; Jones, A.P.; Micelotta, E.R.; Groenewegen, M.A.T. On the Excitation and Formation of Circumstellar Fullerenes. Astrophys. J. 2012, 757, 41. [CrossRef]

40. Peeters, E.; Tielens, A.G.G.M.; Allamandola, L.J.; Wolfire, M.G. The 15-20 $\mu \mathrm{m}$ Emission in the Reflection Nebula NGC 2023. Astrophys. J. 2012, 747, 44. [CrossRef] 
41. Boersma, C.; Rubin, R.H.; Allamandola, L.J. Spatial Analysis of the Polycyclic Aromatic Hydrocarbon Features Southeast of the Orion Bar. Astrophys. J. 2012, 753, 168. [CrossRef]

42. Berné, O.; Tielens, A.G.G.M. Formation of buckminsterfullerene $\left(\mathrm{C}_{60}\right)$ in interstellar space. Proc. Natl. Acad. Sci. USA 2012, 109, 401-406. [CrossRef] [PubMed]

43. Roberts, K.R.G.; Smith, K.T.; Sarre, P.J. Detection of $\mathrm{C}_{60}$ in embedded young stellar objects, a Herbig Ae/Be star and an unusual post-asymptotic giant branch star. Mon. Not. R. Astron. Soc. 2012, 421, 3277-3285. [CrossRef]

44. Otsuka, M.; Kemper, F.; Hyung, S.; Sargent, B.A.; Meixner, M.; Tajitsu, A.; Yanagisawa, K. The Detection of $\mathrm{C}_{60}$ in the Well-characterized Planetary Nebula M1-11. Astrophys. J. 2013, 764, 77. [CrossRef]

45. García-Hernández, D.A.; Górny, S.K. Chemical abundances in Galactic planetary nebulae with Spitzer spectra. Astron. Astrophys. 2014, 567, A12. [CrossRef]

46. Otsuka, M.; Kemper, F.; Cami, J.; Peeters, E.; Bernard-Salas, J. Physical properties of fullerene-containing Galactic planetary nebulae. Mon. Not. R. Astron. Soc. 2014, 437, 2577-2593. [CrossRef]

47. Sloan, G.C.; Lagadec, E.; Zijlstra, A.A.; Kraemer, K.E.; Weis, A.P.; Matsuura, M.; Volk, K.; Peeters, E.; Duley, W.W.; Cami, J.; et al. Carbon-rich Dust Past the Asymptotic Giant Branch: Aliphatics, Aromatics, and Fullerenes in the Magellanic Clouds. Astrophys. J. 2014, 791, 28. [CrossRef]

48. Omont, A. Interstellar fullerene compounds and diffuse interstellar bands. Astron. Astrophys. 2016, 590, A52. [CrossRef]

49. Otsuka, M. Physical properties of the fullerene $\mathrm{C}_{60}$-containing planetary nebula SaSt2-3. Mon. Not. R. Astron. Soc. 2019, 482, 2354-2373. [CrossRef]

50. Campbell, E.K.; Holz, M.; Gerlich, D.; Maier, J.P. Laboratory confirmation of $\mathrm{C}_{60}^{+}$as the carrier of two diffuse interstellar bands. Nature 2015, 523, 322-323. [CrossRef]

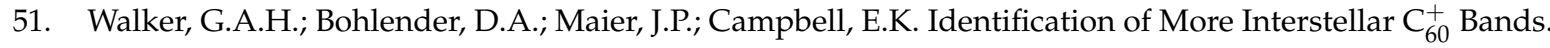
Astrophys. J. Lett. 2015, 812, L8. [CrossRef]

52. Campbell, E.K.; Holz, M.; Maier, J.P.; Gerlich, D.; Walker, G.A.H.; Bohlender, D. Gas Phase Absorption Spectroscopy of $\mathrm{C}_{60}^{+}$and $\mathrm{C}_{70}^{+}$in a Cryogenic Ion Trap: Comparison with Astronomical Measurements. Astrophys. J. 2016, 822, 17. [CrossRef]

53. Campbell, E.K.; Holz, M.; Maier, J.P. Gas-phase Absorption of $C_{70}^{2+}$ below $10 \mathrm{~K}$ : Astronomical Implications. Astrophys. J. 2017, 835, 221. [CrossRef]

54. Campbell, E.K.; Maier, J.P. Isomeric and Isotopic Effects on the Electronic Spectrum of $\mathrm{C}_{60}^{+}$-He: Consequences for Astronomical Observations of $\mathrm{C}_{60}^{+}$. Astrophys. J. 2018, 858, 36. [CrossRef]

55. Strelnikov, D.; Kern, B.; Kappes, M.M. On observing $\mathrm{C}_{60}^{+}$and $\mathrm{C}_{60}^{2+}$ in laboratory and space. Astron. Astrophys. 2015, 584, A55. [CrossRef]

56. Castellanos, P.; Berné, O.; Sheffer, Y.; Wolfire, M.G.; Tielens, A.G.G.M. C 60 in Photodissociation Regions. Astrophys. J. 2014, 794, 83. [CrossRef]

57. Berné, O.; Cox, N.L.J.; Mulas, G.; Joblin, C. Detection of buckminsterfullerene emission in the diffuse interstellar medium. Astron. Astrophys. 2017, 605, L1. [CrossRef]

58. Stecher, T.P.; Donn, B. On Graphite and Interstellar Extinction. Astrophys. J. 1965, 142, 1681. [CrossRef]

59. Fitzpatrick, E.L.; Massa, D. An Analysis of the Shapes of Interstellar Extinction Curves. V. The IR-through-UV Curve Morphology. Astrophys. J. 2007, 663, 320-341. [CrossRef]

60. Clayton, G.C.; Gordon, K.D.; Bianchi, L.C.; Massa, D.L.; Fitzpatrick, E.L.; Bohlin, R.C.; Wolff, M.J. New Ultraviolet Extinction Curves for Interstellar Dust in M31. Astrophys. J. 2015, 815, 14. [CrossRef]

61. Fitzpatrick, E.L.; Massa, D.; Gordon, K.D.; Bohlin, R.; Clayton, G.C. An Analysis of the Shapes of Interstellar Extinction Curves. VII. Milky Way Spectrophotometric Optical-through-Ultraviolet Extinction and Its R-Dependence. arXiv 2019, arXiv:1910.08852.

62. Draine, B.T. Graphite Revisited. Astrophys. J. 2016, 831, 109. [CrossRef]

63. Donn, B.; Wickramasinghe, N.C.; Hudson, J.; Stecher, T.P. On the Formation of Graphite Grains in Cool Stars. Astrophys. J. 1968, 153, 451. [CrossRef]

64. Wickramasinghe, N.C. Interstellar Extinction by Graphite, Iron and Silicate Grains. Nature 1970, $227,51-53$. [CrossRef] [PubMed]

65. Czyzak, S.J.; Santiago, J.J. On the Presence of Graphite in the Interstellar Medium. Astrophys. Space Sci. 1973, 23, 443-458. [CrossRef] 
66. Wickramasinghe, N.C.; Nandy, K. The $2200 \AA$ Axtinction Feature and the Shape-Distribution of Graphite Grains. Astrophys. Space Sci. 1974, 26, 123-129. [CrossRef]

67. Blanco, A.; Bussoletti, E. Graphite grains, carbon depletion and the 2200 A feature. Astrophys. Space Sci. 1981, 78, 467-471. [CrossRef]

68. Draine, B.T.; Malhotra, S. On Graphite and the 2175 Angstrom Extinction Profile. Astrophys. J. 1993, 414, 632. [CrossRef]

69. Duley, W.W.; Seahra, S. Graphite, Polycyclic Aromatic Hydrocarbons, and the 2175 A Extinction Feature. Astrophys. J. 1998, 507, 874-888. [CrossRef]

70. Sakata, A.; Wada, S.; Okutsu, Y.; Shintani, H.; Nakada, Y. Does a $2200 \AA$ hump observed in an artificial carbonaceous composite account for UV interstellar extinction? Nature 1983, 301, 493-494. [CrossRef]

71. Fink, J.; Müller-Heinzerling, T.; Pflüger, J.; Scheerer, B.; Dischler, B.; Koidl, P.; Bubenzer, A.; Sah, R.E. Investigation of hydrocarbon-plasma-generated carbon films by electron-energy-loss spectroscopy. Phys. Rev. B 1984, 30, 4713-4718. [CrossRef]

72. Colangeli, L.; Mennella, V.; Blanco, A.; Fonti, S.; Bussoletti, E.; Gumlich, H.E.; Mertins, H.C.; Jung, C. Extreme-Ultraviolet Extinction Measurements on Hydrogenated and Dehydrogenated Amorphous Carbon Grains. Astrophys. J. 1993, 418, 435. [CrossRef]

73. Sakata, A.; Wada, S.; Tokunaga, A.T.; Narisawa, T.; Nakagawa, H.; Ono, H. Ultraviolet Spectra of Quenched Carbonaceous Composite Derivatives: Comparison to the "217 Nanometer" Interstellar Absorption Feature. Astrophys. J. 1994, 430, 311. [CrossRef]

74. Mennella, V.; Colangeli, L.; Palumbo, P.; Rotundi, A.; Schutte, W.; Bussoletti, E. Activation of an Ultraviolet Resonance in Hydrogenated Amorphous Carbon Grains by Exposure to Ultraviolet Radiation. Astrophys. J. Lett. 1996, 464, L191. [CrossRef]

75. Zubko, V.G.; Mennella, V.; Colangeli, L.; Bussoletti, E. Optical constants of cosmic carbon analogue grains-I. Simulation of clustering by a modified continuous distribution of ellipsoids. Mon. Not. R. Astron. Soc. 1996, 282, 1321-1329. [CrossRef]

76. Schnaiter, M.; Mutschke, H.; Dorschner, J.; Henning, T.; Salama, F. Matrix-isolated Nano-sized Carbon Grains as an Analog for the 217.5 Nanometer Feature Carrier. Astrophys. J. 1998, 498, 486-496. [CrossRef]

77. Jäger, C.; Krasnokutski, S.; Staicu, A.; Huisken, F.; Mutschke, H.; Henning, T.; Poppitz, W.; Voicu, I. Identification and Spectral Properties of Polycyclic Aromatic Hydrocarbons in Carbonaceous Soot Produced by Laser Pyrolysis. Astrophys. J. Suppl. Ser. 2006, 166, 557-566. [CrossRef]

78. Jäger, C.; Mutschke, H.; Henning, T.; Huisken, F. Spectral Properties of Gas-phase Condensed Fullerene-like Carbon Nanoparticles from Far-ultraviolet to Infrared Wavelengths. Astrophys. J. 2008, 689, 249-259. [CrossRef]

79. Steglich, M.; Jäger, C.; Rouillé, G.; Huisken, F.; Mutschke, H.; Henning, T. Electronic Spectroscopy of Medium-sized Polycyclic Aromatic Hydrocarbons: Implications for the Carriers of the $2175 \AA$ AV Bump. Astrophys. J. Lett. 2010, 712, L16-L20. [CrossRef]

80. Duley, W.W.; Hu, A. The $217.5 \mathrm{~nm}$ Band, Infrared Absorption, and Infrared Emission Features in Hydrogenated Amorphous Carbon Nanoparticles. Astrophys. J. 2012, 761, 115. [CrossRef]

81. Bescond, A.; Yon, J.; Ouf, F.-X.; Rozé, C.; Coppalle, A.; Parent, P.; Ferry, D.; Laffon, C. Soot optical properties determined by analyzing extinction spectra in the visible near-UV: Toward an optical speciation according to constituents and structure. J. Aerosol Sci. 2016, 101, 118-132. [CrossRef]

82. Gavilan, L.; Le, K.C.; Pino, T.; Alata, I.; Giuliani, A.; Dartois, E. Polyaromatic disordered carbon grains as carriers of the UV bump: Far-UV to mid-IR spectroscopy of laboratory analogs. Astron. Astrophys. 2017, 607, A73. [CrossRef]

83. Draine, B.T.; Lee, H.M. Optical Properties of Interstellar Graphite and Silicate Grains. Astrophys. J. 1984, 285, 89. [CrossRef]

84. Huss, G.R.; Meshik, A.P.; Smith, J.B.; Hohenberg, C.M. Presolar diamond, silicon carbide, and graphite in carbonaceous chondrites: Implications for thermal processing in the solar nebula. Geochim. Cosmochim. Acta 2003, 67, 4823-4848. [CrossRef]

85. Anders, E.; Zinner, E. Interstellar Grains in Primitive Meteorites: Diamond, Silicon Carbide, and Graphite. Meteoritics 1993, 28, 490. [CrossRef] 
86. Matsuura, M.; Sloan, G.C.; Zijlstra, A.A.; Wood, P.R.; Harris, J.G.; Bernard-Salas, J.; van Loon, J.T.; Whitelock, P.A.; Menzies, J.W. Infrared Molecular Bands of Carbon-Rich Stars in Nearby Galaxies. In Why Galaxies Care about AGB Stars: Their Importance as Actors and Probes, Proceedings of the ASP Conference, Vienna, Austria, 7-11 August 2006; Astronomical Society of the Pacific: San Francisco, CA, USA, 2007; Volume 378, p. 450.

87. Gruendl, R.A.; Chu, Y.-H.; Seale, J.P.; Matsuura, M.; Speck, A.K.; Sloan, G.C.; Looney, L.W. Discovery of Extreme Carbon Stars in the Large Magellanic Cloud. Astrophys. J. Lett. 2008, 688, L9. [CrossRef]

88. Speck, A.K.; Corman, A.B.; Wakeman, K.; Wheeler, C.H.; Thompson, G. Silicon Carbide Absorption Features: Dust Formation in the Outflows of Extreme Carbon Stars. Astrophys. J. 2009, 691, 1202-1221. [CrossRef]

89. Yang, X.; Chen, P.; He, J. Molecular and dust features of $29 \mathrm{SiC}$ carbon AGB stars. Astron. Astrophys. 2004, 414, 1049-1063. [CrossRef]

90. Whittet, D.C.B.; Duley, W.W.; Martin, P.G. On the abundance of silicon carbide in the interstellar medium.. Mon. Not. R. Astron. Soc. 1990, 244, 427.

91. Min, M.; Waters, L.B.F.M.; de Koter, A.; Hovenier, J.W.; Keller, L.P.; Markwick-Kemper, F. The shape and composition of interstellar silicate grains. Astron. Astrophys. 2007, 462, 667-676. [CrossRef]

92. Rogantini, D.; Costantini, E.; Zeegers, S.T.; de Vries, C.P.; Mehdipour, M.; de Groot, F.; Mutschke, H.; Psaradaki, I.; Waters, L.B.F.M. Interstellar dust along the line of sight of GX 3+1. Astron. Astrophys. 2019, 630, A143. [CrossRef]

93. Leger, A.; Puget, J.L. Identification of the "unidentified" IR emission features of interstellar dust?. Astron. Astrophys. 1984, 500, 279-282.

94. Allamandola, L.J.; Tielens, A.G.G.M.; Barker, J.R. Polycyclic aromatic hydrocarbons and the unidentified infrared emission bands: Auto exhaust along the milky way. Astrophys. J. Lett. 1985, 290, L25-L28. [CrossRef]

95. Peeters, E.; Hony, S.; van Kerckhoven, C.; Tielens, A.G.G.M.; Allamandola, L.J.; Hudgins, D.M.; Bauschlicher, C.W. The rich 6 to $9 \mathrm{vec}$ mu m spectrum of interstellar PAHs. Astron. Astrophys. 2002, 390, 1089-1113. [CrossRef]

96. Sloan, G.C.; Jura, M.; Duley, W.W.; Kraemer, K.E.; Bernard-Salas, J.; Forrest, W.J.; Sargent, B.; Li, A.; Barry, D.J.; Bohac, C.J.; et al. The Unusual Hydrocarbon Emission from the Early Carbon Star HD 100764: The Connection between Aromatics and Aliphatics. Astrophys. J. 2007, 664, 1144-1153. [CrossRef]

97. Evans, A.; Rawlings, J.M.C. 'PAH' emission in novae.. Mon. Not. R. Astron. Soc. 1994, 269, $427-434$. [CrossRef]

98. Boersma, C.; Bouwman, J.; Lahuis, F.; van Kerckhoven, C.; Tielens, A.G.G.M.; Waters, L.B.F.M.; Henning, T. The characteristics of the IR emission features in the spectra of Herbig Ae stars: Evidence for chemical evolution. Astron. Astrophys. 2008, 484, 241-249. [CrossRef]

99. Keller, L. D.; Sloan, G. C.; Forrest, W. J.; Ayala, S.; D’Alessio, P.; Shah, S.; Calvet, N.; Najita, J.; Li, A.; Hartmann, L.; et al. PAH Emission from Herbig Ae/Be Stars. Astrophys. J. 2008, 684, 411-429. [CrossRef]

100. Acke, B.; Bouwman, J.; Juhász, A.; Henning, T.; van den Ancker, M.E.; Meeus, G.; Tielens, A.G.G.M.; Waters, L.B.F.M. Spitzer's View on Aromatic and Aliphatic Hydrocarbon Emission in Herbig Ae Stars. Astrophys. J. 2010, 718, 558-574. [CrossRef]

101. Pino, T.; Dartois, E.; Cao, A.-T.; Carpentier, Y.; Chamaillé, T.; Vasquez, R.; Jones, A.P.; D’Hendecourt, L.; Bréchignac, P. The $6.2 \mu \mathrm{m}$ band position in laboratory and astrophysical spectra: A tracer of the aliphatic to aromatic evolution of interstellar carbonaceous dust. Astron. Astrophys. 2008, 490, 665-672. [CrossRef]

102. Carpentier, Y.; Féraud, G.; Dartois, E.; Brunetto, R.; Charon, E.; Cao, A.-T.; d’Hendecourt, L.; Bréchignac, P.; Rouzaud, J.-N.; Pino, T. Nanostructuration of carbonaceous dust as seen through the positions of the 6.2 and $7.7 \mu \mathrm{m}$ AIBs. Astron. Astrophys. 2012, 548, A40. [CrossRef]

103. Gadallah, K.A.K.; Mutschke, H.; Jäger, C. Analogs of solid nanoparticles as precursors of aromatic hydrocarbons. Astron. Astrophys. 2013, 554, A12. [CrossRef]

104. Volk, K.; Kwok, S.; Hrivnak, B.; Szczerba, R. ISO Results for Protoplanetary Nebulae. Astrophys. Space Sci. Libr. 2001, 265, 323.

105. Hony, S.; Waters, L.B.F.M.; Tielens, A.G.G.M. The carrier of the " 30 " mu m emission feature in evolved stars. A simple model using magnesium sulfide. Astron. Astrophys. 2002, 390, 533-553. [CrossRef]

106. Gauba, G.; Parthasarathy, M. Circumstellar dust shells of hot post-AGB stars. Astron. Astrophys. 2004, 417, 201-215. [CrossRef] 
107. Chen, P.-S.; Yang, X.-H.; Shan, H.-G. Infrared spectral evolution of carbon stars. Res. Astron. Astrophys. 2010, 10, 363-382. [CrossRef]

108. Sorrell, W.H. Annealed HAC mantles in diffuse dust clouds.. Mon. Not. R. Astron. Soc. 1991, $248,439$. [CrossRef]

109. Sorrell, W.H. The lambda 2175-A feature from irradiated graphitic particles. Mon. Not. R. Astron. Soc. 1990, 243, 570-587.

110. Hecht, J.H. The Nature of the Dust around R Coronae Borealis Stars: Isolated Amorphous Carbon or Graphite Fractals? Astrophys. J. 1991, 367, 635. [CrossRef]

111. Mennella, V. Activation Energy of CH Bond Formation in Carbon Grains Irradiated with Hydrogen Atoms. Astrophys. J. Lett. 2006, 647, L49-L52. [CrossRef]

112. Mennella, V.H. Atom Irradiation of Carbon Grains under Simulated Dense Interstellar Medium Conditions: The Evolution of Organics from Diffuse Interstellar Clouds to the Solar System. Astrophys. J. 2010, 718, 867-875. [CrossRef]

113. Allen, D.A.; Wickramasinghe, D.T. Diffuse interstellar absorption bands between 2.9 and 4.0 microns. Nature 1981, 294, 239. [CrossRef]

114. Duley, W.W.; Williams, D.A. A 3.4 Mu-m absorption band in amorphous carbon : Implications for interstellar dust.. Mon. Not. R. Astron. Soc. 1983, 205, 67P-70. [CrossRef]

115. Greenberg, J.M.; Li, A.; Mendoza-Gomez, C.X.; Schutte, W.A.; Gerakines, P.A.; de Groot, M. Approaching the Interstellar Grain Organic Refractory Component. Astrophys. J. Lett. 1995, 455, L177. [CrossRef]

116. Dartois, E.; Marco, O.; Muñoz-Caro, G.M.; Brooks, K.; Deboffle, D.; d'Hendecourt, L. Organic matter in Seyfert 2 nuclei: Comparison with our Galactic center lines of sight. Astron. Astrophys. 2004, 423, 549-558. [CrossRef]

117. Chiar, J.E.; Tielens, A.G.G.M.; Adamson, A.J.; Ricca, A. The Structure, Origin, and Evolution of Interstellar Hydrocarbon Grains. Astrophys. J. 2013, 770, 78. [CrossRef]

118. Mason, R.E.; Wright, G.; Pendleton, Y.; Adamson, A. Hydrocarbon Dust Absorption in Seyfert Galaxies and Ultraluminous Infrared Galaxies. Astrophys. J. 2004, 613, 770-780. [CrossRef]

119. Risaliti, G.; Maiolino, R.; Marconi, A.; Sani, E.; Berta, S.; Braito, V.; della Ceca, R.; Franceschini, A.; Salvati, M. Unveiling the nature of Ultraluminous Infrared Galaxies with $3-4 \mu \mathrm{m}$ spectroscopy*. Mon. Not. R. Astron. Soc. 2006, 365, 303-320. [CrossRef]

120. Imanishi, M.; Nakagawa, T.; Shirahata, M.; Ohyama, Y.; Onaka, T. AKARI IRC Infrared 2.5-5 $\mu \mathrm{m}$ Spectroscopy of a Large Sample of Luminous Infrared Galaxies. Astrophys. J. 2010, 721, 1233-1261. [CrossRef]

121. Pety, J.; Teyssier, D.; Fossé, D.; Gerin, M.; Roueff, E.; Abergel, A.; Habart, E.; Cernicharo, J. Are PAHs precursors of small hydrocarbons in photo-dissociation regions? The Horsehead case. Astron. Astrophys. 2005, 435, 885-899. [CrossRef]

122. Pety, J.; Gratier, P.; Guzmán, V.; Roueff, E.; Gerin, M.; Goicoechea, J.R.; Bardeau, S.; Sievers, A.; Le Petit, F.; Le Bourlot, J.; et al. The IRAM-30 m line survey of the Horsehead PDR. II. First detection of the $1-\mathrm{C}_{3} \mathrm{H}^{+}$ hydrocarbon cation. Astron. Astrophys. 2012, 548, A68. [CrossRef]

123. Guzmán, V.V.; Goicoechea, J.R.; Pety, J.; Gratier, P.; Gerin, M.; Roueff, E.; Le Petit, F.; Le Bourlot, J.; Faure, A. The IRAM-30 m line survey of the Horsehead PDR-IV. Comparative chemistry of $\mathrm{H}_{2} \mathrm{CO}$ and $\mathrm{CH}_{3} \mathrm{OH}$. Astron. Astrophys. 2013, 560, A73. [CrossRef]

124. Gratier, P.; Pety, J.; Guzmán,V.; Gerin, M.; Goicoechea, J.R.; Roueff, E.; Faure, A. The IRAM-30 m line survey of the Horsehead PDR-III. High abundance of complex (iso-)nitrile molecules in UV-illuminated gas. Astron. Astrophys. 2013, 557, A101. [CrossRef]

125. Guzmán, V.V.; Pety, J.; Goicoechea, J.R.; Gerin, M.; Roueff, E.; Gratier, P.; Öberg, K.I. Spatially Resolved $\mathrm{L}_{-} \mathrm{C}_{3} \mathrm{H}^{+}$Emission in the Horsehead Photodissociation Region: Further Evidence for a Top-Down Hydrocarbon Chemistry. Astrophys. J. Lett. 2015, 800, L33. [CrossRef]

126. Dartois, E.; Chabot, M.; Pino, T.; Béroff, K.; Godard, M.; Severin, D.; Bender, M.; Trautmann, C. Swift heavy ion irradiation of interstellar dust analogues. Small carbonaceous species released by cosmic rays. Astron. Astrophys. 2017, 599, A130. [CrossRef]

127. Alata, I.; Jallat, A.; Gavilan, L.; Chabot, M.; Cruz-Diaz, G.A.; Caro, G.M.M.; Béroff, K.; Dartois, E. Vacuum ultraviolet of hydrogenated amorphous carbons. II. Small hydrocarbons production in Photon Dominated Regions. Astron. Astrophys. 2015, 584, A123 [CrossRef] 
128. Mennella, V.; Muñoz Caro, G.M.; Ruiterkamp, R.; Schutte, W.A.; Greenberg, J.M.; Brucato, J.R.; Colangeli, L. $\mathrm{UV}$ photodestruction of $\mathrm{CH}$ bonds and the evolution of the $3.4 \mathrm{mu} \mathrm{m}$ feature carrier. II. The case of hydrogenated carbon grains. Astron. Astrophys. 2001, 367, 355-361. [CrossRef]

129. Mennella, V.; Baratta, G.A.; Esposito, A.; Ferini, G.; Pendleton, Y.J. The Effects of Ion Irradiation on the Evolution of the Carrier of the 3.4 Micron Interstellar Absorption Band. Astrophys. J. 2003, 587, 727-738. [CrossRef]

130. Alata, I.; Cruz-Diaz, G.A.; Muñoz Caro, G.M.; Dartois, E. Vacuum ultraviolet photolysis of hydrogenated amorphous carbons. I. Interstellar $\mathrm{H}_{2}$ and $\mathrm{CH}_{4}$ formation rates. Astron. Astrophys. 2014, 569, A119. [CrossRef]

131. Duley, W.W.; Zaidi, A.; Wesolowski, M.J.; Kuzmin, S. Small molecules from the decomposition of interstellar carbons. Mon. Not. R. Astron. Soc. 2015, 447, 1242-1246. [CrossRef]

132. Maté, B.; Molpeceres, G.; Jiménez-Redondo, M.; Tanarro, I.; Herrero, V.J. High-energy Electron Irradiation of Interstellar Carbonaceous Dust Analogs: Cosmic-ray Effects on the Carriers of the $3.4 \mu \mathrm{m}$ Absorption Band. Astrophys. J. 2016, 831, 51. [CrossRef] [PubMed]

133. Pino, T.; Chabot, M.; Béroff, K.; Godard, M.; Fernandez-Villoria, F.; Le, K.C.; Breuer, L.; Herder, M.; Wucher, A.; Bender, M.; et al. Release of large polycyclic aromatic hydrocarbons and fullerenes by cosmic rays from interstellar dust. Swift heavy ion irradiations of interstellar carbonaceous dust analogue. Astron. Astrophys. 2019, 623, A134. [CrossRef]

134. Léger, A.; D’Hendecourt, L.; Verstraete, L.; Joblin, C. Polycyclic Aromatic Hydrocarbons (PAH's): Very Abundant Organic Molecules in the Interstellar Medium. Bioastronomy 1991, 390, 88-92.

135. Draine, B.T.; Li, A. Infrared Emission from Interstellar Dust. IV. The Silicate-Graphite-PAH Model in the Post-Spitzer Era. Astrophys. J. 2007, 657, 810-837. [CrossRef]

136. Dwek, E.; Galliano, F.; Jones, A. The Cycle of Dust in the Milky Way: Clues from the High-Redshift and Local Universe. Cosmic Dust Near Far 2009, 414, 183.

137. Galliano, F.; Dwek, E.; Chanial, P. Stellar Evolutionary Effects on the Abundances of Polycyclic Aromatic Hydrocarbons and Supernova-Condensed Dust in Galaxies. Astrophys. J. 2008, 672, 214-243. [CrossRef]

138. Compiègne, M.; Flagey, N.; Noriega-Crespo, A.; Martin, P.G.; Bernard, J.-P.; Paladini, R.; Molinari, S. Dust in the Diffuse Emission of the Galactic Plane: The Herschel/Spitzer Spectral Energy Distribution Fitting. Astrophys. J. Lett. 2010, 724, L44-L47. [CrossRef]

139. Gredel, R.; Carpentier, Y.; Rouillé, G.; Steglich, M.; Huisken, F.; Henning, T. Abundances of PAHs in the ISM: Confronting observations with experimental results. Astron. Astrophys. 2011, 530, A26. [CrossRef]

140. Khramtsova, M.S.; Wiebe, D.S.; Boley, P.A.; Pavlyuchenkov, Y.N. Polycyclic aromatic hydrocarbons in spatially resolved extragalactic star-forming complexes. Mon. Not. R. Astron. Soc. 2013, 431, 2006-2016. [CrossRef]

141. Ciesla, L.; Boquien, M.; Boselli, A.; Buat, V.; Cortese, L.; Bendo, G.J.; Heinis, S.; Galametz, M.; Eales, S.; Smith, M.W.L.; et al. Dust spectral energy distributions of nearby galaxies: An insight from the Herschel Reference Survey. Astron. Astrophys. 2014, 565, A128. [CrossRef]

142. Seok, J.Y.; Hirashita, H.; Asano, R.S. Formation history of polycyclic aromatic hydrocarbons in galaxies. Mon. Notices R. Astron. Soc. 2014, 439, 2186-2196. [CrossRef]

143. Sandford, S.A.; Pendleton, Y.J.; Allamandola, L.J. The Galactic Distribution of Aliphatic Hydrocarbons in the Diffuse Interstellar Medium. Astrophys. J. 1995, 440, 697. [CrossRef] [PubMed]

144. Pendleton, Y.J.; Sandford, S.A.; Allamandola, L.J.; Tielens, A.G.G.M.; Sellgren, K. Near-Infrared Absorption Spectroscopy of Interstellar Hydrocarbon Grains. Astrophys. J. 1994, 437, 683. [CrossRef]

145. Duley, W.W. Infrared Absorption Due to Hydrogenated Amorphous Carbon in the Diffuse Interstellar Medium. Astrophys. J. Lett. 1994, 430, L133. [CrossRef]

146. Duley, W.W.; Scott, A.D.; Seahra, S.; Dadswell, G. Integrated Absorbances in the $3.4 \mu \mathrm{m} \mathrm{CH}_{n} \mathrm{Band}$ in Hydrogenated Amorphous Carbon. Astrophys. J. Lett. 1998, 503, L183-L185. [CrossRef]

147. Zubko, V.; Dwek, E.; Arendt, R.G. Interstellar Dust Models Consistent with Extinction, Emission, and Abundance Constraints. Astrophys. J. Suppl. Ser. 2004, 152, 211-249. [CrossRef] 
148. Draine, B.T.; Dale, D.A.; Bendo, G.; Gordon, K.D.; Smith, J.D.T.; Armus, L.; Engelbracht, C.W.; Helou, G.; Kennicutt, R.C., Jr.; Li, A.; et al. Dust Masses, PAH Abundances, and Starlight Intensities in the SINGS Galaxy Sample. Astrophys. J. 2007, 663, 866-894. [CrossRef]

149. Jones, A.P.; Köhler, M.; Ysard, N.; Bocchio, M.; Verstraete, L. The global dust modelling framework THEMIS. Astron. Astrophys. 2017, 602, A46. [CrossRef]

(C) 2019 by the authors. Licensee MDPI, Basel, Switzerland. This article is an open access article distributed under the terms and conditions of the Creative Commons Attribution (CC BY) license (http:/ / creativecommons.org/licenses/by/4.0/). 